\title{
PREPARATION AND CHARACTERIZATION OF SOLID DISPERSION OF MODAFINIL USING PEG6000 AS HYDROPHILIC CARRIER FOR IMPROVEMENT OF DISSOLUTION PROFILE
}

\author{
Vyas JR ${ }^{\mathbf{a}^{*}}$, Kathiriya PR ${ }^{\mathrm{b}}$, Upadhyay $\mathrm{UM}^{\mathrm{b}}{ }^{\mathrm{b}}$, Patel $\mathrm{JK}^{\mathrm{c}}$, \\ a, Department of Pharmaceutics, Bhagwant University, Ajmer, Rajasthan, India. 305004 \\ b, Sigma Institute of Pharmacy, Bakrol, Baroda. Gujarat, India. 390022 \\ c, Nootan Pharmacy College, Visnagar, Gujarat, India. 384315
}

\begin{abstract}
Solid dispersions of modafinil were prepared using polyethyleneglycols, in various proportions by melting, solvent evaporation and kneading method. Based on the solubility, PEG6000 was selected and solid dispersion batch F6 containing drug:PEG6000 in 1:3 ration was formulated as tablet (batch TF6) and evaluated for in-vitro drug dissolution and six month stability; and the results were compared with that of tablet containing physical mixture of drug:PEG6000 in 1:3 ratio (batch TF24) and convention tablet (batch CT) containing pure drug. Tablet batch TF24 had shown significant improvement of dissolution profile of modafinil when compared with that of tablet batch TF6 and CT. Present study conclusively demonstrated that PEG6000 enhanced water solubility of modafinil by amorphisation, which was confirmed XRD and DSC; and also the melting method was found better than solvent evaporation.

Key-words: solid dispersion, polyethyleneglycols, modafinil, solubility enhancement, in vitro dissolution, characterization.
\end{abstract}

\section{INTRODUCTION}

Many potential drug candidates are characterized by a low oral bioavailability. Often, poor drug dissolution/solubility rather than limited permeation through the epithelia of the gastrointestinal tract are responsible for low oral bioavailability. The relationship between solution rate and absorption is particularly distinct when considering drugs of low solubility. Consequently, numerous attempts have been made to modify the dissolution characteristics of certain drugs in an effort to attain more rapid and more complete absorption. Among the techniques to increase aqueous solubility/dissolution rate, the formulation of solid dispersions is one of the most popular ones ${ }^{1-2}$, although few marketed products rely on this concept. The interest in amorphous drug-polymer solid dispersions has grown due to the potential of improving bioavailability, particularly for poorly water-soluble drugs ${ }^{3-7}$. The basis for this interest stems from the increased rate of dissolution, which can range from hundreds to thousands fold increase, even for the most insoluble active pharmaceutical ingredients ${ }^{3}$. For drugs whose bioavailability is limited due to poor aqueous solubility (as in BSC class II drugs), the improvement in solubility may lead to enhanced bioavailability ${ }^{8-11}$. Solid dispersion represents a useful pharmaceutical technique for increasing the dissolution, absorption, and therapeutic efficacy of drugs in dosage forms ${ }^{12-13}$. The properties, performance, and practical applications of solid dispersions depend on factors such as: (a) the method of preparation, (b) composition, (c) selection of a suitable carrier, and (d) physicochemical properties of the drug ${ }^{1}$, 14

Modafinil is approved by the USFDA for the treatment of narcolepsy, hypersomnia, shift work sleep disorder and excessive daytime sleepiness associated with obstructive sleep apnoea and in adult it is used in attention deficient /hyperactivity disorder (ADHD) ${ }^{15}$. It is rapidly absorbed after oral administration with peak plasma concentrations occurring at 2-4 hours. But the oral bioavailability the drug is very poor due to water insolubility ${ }^{15}$. Modafinil is BCS class II drug; hence improvement of dissolution will lead to enhancement of bioavailability.

In the present study, we prepared solid dispersions of modafinil using hydrophilic carriers (polyethyleneglycols) by two different methods i.e. melting method and solvent evaporation method, to improve its solubility. For purposes of comparison, physical mixtures were prepared by simple mixing and homogenization after pulverization of drug and carriers.

*Corresponding author:

Jigar R. Vyas, Associate Professor, Sigma Institute of Pharmacy, Bakrol, Baroda, Gujarat, India. 390022. Tel.: +919428764734,E-mail:drjrvyas@yahoo.co.in 


\section{MATERIALS}

Modafinil was obtained as a gift sample from alembic pharmaceuticals Ltd. (Vadodara, India); PEG4000, PEG6000, PEG8000, acetone and chloroform were purchased from S.D.Fine Chemicals Ltd. (Mumbai, India); hydrochloric acid was purchased from LobaChem (Mumbai, India). Distilled water was freshly prepared and used for the study. All the chemicals and reagents were of analytical reagent (AR) grade and used without further purifications.

\section{METHODS}

\section{Preparation of solid dispersions}

Solid dispersions were prepared by three different methods i.e. melting method, solvent evaporation method and physical mixture.

a) Melting method: Solid dispersions containing different weight ratios (1:1, 1:2, 1:3) of drug in PEG4000, PEG6000, and PEG8000 were prepared by melting the carriers in porcelain dish (at around $10^{\circ} \mathrm{C}$ more than the melting point of carriers on sand bath), dispersing the drug onto the molten carrier and cooling immediately on freezing mixture of ice and sodium chloride. The solid dispersions were then allowed to cool at an ambient temperature and stored in desiccators for 24 hours. The dry mass was scrapped, crushed and ground in a mortar and passed through sieve \#40. The dried mass was stored in desiccator until further use.

b) Solvent evaporation method: Solid dispersions containing different weight ratios $(1: 1,1: 2,1: 3)$ of drug in PEG4000, PEG6000, and PEG8000 were prepared by dissolving required amount of drug and carriers in solvent system containing acetone and chloroform in 1:1 proportion. The solvent was evaporated at $40^{\circ} \mathrm{C}$ on water bath with continuous stirring and the resulting residues were dried under vacuum for 3 hours and stored in desiccators for overnight. The dry mass was ground in a mortar, passed through sieve \#40 and stored in desiccator until further use.

c) Kneading: Drug and carriers were blended in desired proportions using spatula for 10 minutes and then ground in mortar with pestle. The co-grinding mixture was then passed through sieve \#40 and stored in desiccator until further use.

\section{Evaluation of solid dispersions}

a) Saturation solubility: The saturation solubility of drug, carriers and all the solid dispersions was determined by dispersing $1 \mathrm{~g}$ of drug, carrier or solid dispersion into $100 \mathrm{ml}$ of distilled water contained in glass bottle and shaken for not less than 24 hours. Solubility was then determined in $\mathrm{mg} / \mathrm{ml}$ using spectrophotometer with $\lambda \max$ at $222 \mathrm{~nm}$ after filtration (through a $0.45 \mu \mathrm{m}$ Millipore filter) and necessary dilutions. b) Melting point: Melting point of drug, polymer and all the solid dispersions was determined using precision melting point apparatus. Two samples were tested at a time by placing a pinch of sample into the capillary and heating at slow and consistent rate.

c) Stability: The prepared solid dispersions were stored in stability chamber at $45 \pm 2^{\circ} \mathrm{C}$ with $75 \% \mathrm{RH}$ for 6 months. The formulations were analysed for one point in vitro dissolution, solubility and melting point after 6 months and compared with the results obtained with formulations prepared and analysed immediately.

\section{Characterisation of solid dispersions}

a) Differential Scanning Calorimetry (DSC) Analysis: DSC scans of the powdered samples were recorded using DSC- 822e Mettler Toledo with the Stare software. All the samples were weighed (4-5 mg) and heated for total time of $40 \mathrm{~min}$ at a scanning rate of $5^{\circ} \mathrm{C} / \mathrm{min}$ under dry air $\left(\mathrm{N}_{2}\right)$ flow $(50 \mathrm{ml} / \mathrm{min})$ at pressure of 25 psi between 50 and $250^{\circ} \mathrm{C}$ (furnace temperature). Aluminium pans and lids $(40 \mu 1$ capacity) were used for the study.

b) X-ray diffraction analysis: X-ray diffraction (XRD) patterns were recorded on an X-diffractometer (Phillip PW 1130/00 diffractometer, Natherland), employing $\mathrm{CuK}_{\infty}$ radiation source operating at $30 \mathrm{~mA}$ and $40 \mathrm{kV}$. Samples were scanned from 6 to $40^{\circ} 2 \theta$ at a scanning rate of $0.02^{\circ} 2 \theta \mathrm{s}^{-1}$.

\section{Preparation of tablets}

Tablets containing either solid dispersions, drug-carrier physical mixture or simple drug equivalent to $100 \mathrm{mg}$ of modafinil were prepared by direct compression method after mixing with required amount of different ingredients as shown in table 4 .

\section{Evaluation of tablets containing modafinil}

All the prepared tablets were subjected to routine quality control tests like hardness, friability and weight variation before evaluating for in vitro dissolution and stability study.

In vitro dissolution study: In vitro dissolution study of modafinil was performed on 8 vessel USP type II dissolution test apparatus in $0.1 \mathrm{~N} \mathrm{HCl}$ with constant temperature $37+2^{\circ} \mathrm{C}$ and speed 50rpm. Aliquots were withdrawn at predetermined time intervals, analyzed by UV-visible spectrophotometric method and cumulative percentage release of drug was recorded.

\section{RESULTS}

Solid dispersions of Modafinil were prepared successfully by physical mixture and solvent evaporation method and compared with melting method of drug and carriers. All the prepared formulations were evaluated for saturation solubility and \%CSE; and the data of composition and evaluations of all the formulations were recorded in Table 1, Table 2 and Table 3. 
Table 1 Composition and evaluations of solid dispersions prepared by melting method

\begin{tabular}{|c|c|c|c|c|c|c|}
\hline Batch & Drug & $\begin{array}{c}\text { PEG } \\
\mathbf{4 0 0 0}\end{array}$ & $\begin{array}{c}\text { PEG } \\
\mathbf{6 0 0 0}\end{array}$ & PEG 8000 & SS & \% CSE \\
\hline F1 & 100 & 100 & - & - & $0.573 \pm 1.09$ & 27.902 \\
\hline F2 & 100 & 200 & - & - & $1.103 \pm 1.12$ & 73.103 \\
\hline F3 & 100 & 300 & - & - & $1.956 \pm 0.98$ & 112.202 \\
\hline F4 & 100 & - & 100 & - & $0.594 \pm 2.12$ & 32.59 \\
\hline F5 & 100 & - & 200 & - & $1.162 \pm 1.67$ & 79.69 \\
\hline F6 & 100 & - & 300 & - & $2.143 \pm 1.90$ & 126.12 \\
\hline F7 & 100 & - & - & 100 & $0.564 \pm 1.43$ & 25.96 \\
\hline F8 & 100 & - & - & 200 & $1.104 \pm 1.02$ & 73.20 \\
\hline F9 & 100 & - & - & 300 & $2.036 \pm 0.78$ & 118.14 \\
\hline
\end{tabular}

$S S=$ Saturation solubility in $\mathrm{mg} / \mathrm{ml} ; \% C S E=$ cum. solubility enhancement

Table 2 Composition and evaluations of solid dispersions prepared by solvent evaporation method

\begin{tabular}{|c|c|c|c|c|c|c|}
\hline Batch & Drug & PEG 4000 & PEG 6000 & PEG 8000 & SS & \% CSE \\
\hline F10 & 100 & 100 & - & - & $0.539 \pm 1.01$ & 20.228 \\
\hline F11 & 100 & 200 & - & - & $1.037 \pm 1.21$ & 65.717 \\
\hline F12 & 100 & 300 & - & - & $1.839 \pm 1.56$ & 103.47 \\
\hline F13 & 100 & - & 100 & - & $0.558 \pm 0.83$ & 24.63 \\
\hline F14 & 100 & - & 200 & - & $1.092 \pm 2.12$ & 71.91 \\
\hline F15 & 100 & - & 300 & - & $2.014 \pm 1.24$ & 116.55 \\
\hline F16 & 100 & - & - & 100 & $0.519 \pm 1.37$ & 15.91 \\
\hline F17 & 100 & - & - & 200 & $1.016 \pm 1.22$ & 63.37 \\
\hline F18 & 100 & - & - & 300 & $1.873 \pm 1.01$ & 106.06 \\
\hline
\end{tabular}

SS=Saturation solubility in $\mathrm{mg} / \mathrm{ml}$; \%CSE=cum. Solubility enhancement

Table 3 Composition and evaluations of physical mixtures

\begin{tabular}{|c|c|c|c|c|c|c|}
\hline Batch & Drug & PEG 4000 & PEG 6000 & PEG 8000 & SS & \% CSE \\
\hline F19 & 100 & 100 & - & - & $0.461 \pm 1.12$ & 2.902 \\
\hline F20 & 100 & 200 & - & - & $0.529 \pm 1.23$ & 9.089 \\
\hline F21 & 100 & 300 & - & - & $0.665 \pm 1.12$ & 16.149 \\
\hline F22 & 100 & - & 100 & - & $0.453 \pm 0.87$ & 1.12 \\
\hline F23 & 100 & - & 200 & - & $0.465 \pm 2.67$ & 1.88 \\
\hline F24 & 100 & - & 300 & - & $0.857 \pm 2.65$ & 30.45 \\
\hline F25 & 100 & - & - & 100 & $0.451 \pm 1.78$ & 0.61 \\
\hline F26 & 100 & - & - & 200 & $0.458 \pm 1.07$ & 1.10 \\
\hline F27 & 100 & - & - & 300 & $0.844 \pm 1.35$ & 29.49 \\
\hline
\end{tabular}

SS=Saturation solubility in $\mathrm{mg} / \mathrm{ml} ; \% \mathrm{CSE}=$ cum. Solubility enhancement

Batch F24 was selected after comparing the saturation solubility, formulated in tablet dosage form and extensively evaluated for in vitro dissolution and stability study. Tablet containing batch F6 and pure drug were also prepared and evaluated to get better comparison of the data.

All the three tablet batches were characterised for \%CSE (Cumulative Solubility Enhancement), saturation solubility and in vitro drug dissolution, as recorded in table 5, to understand stability of the formulations.

Table 4 Composition of tablet containing pure drug, solid dispersion and physical mixture

\begin{tabular}{|l|c|c|c|}
\hline \multirow{2}{*}{ Tablet ingredients } & \multicolumn{3}{|c|}{ Amount in mg } \\
\cline { 2 - 4 } & Batch TF6 & Batch TF24 & Batch CT \\
\hline Drug* & 500 & 500 & 100 \\
\hline Cross povidone XL & 28 & 28 & 28 \\
\hline PVP K30 & 50 & 50 & 50 \\
\hline Corn starch & 15 & 15 & 15 \\
\hline Flow Lac 100 & 80 & 85 & 90 \\
\hline MCC & 27 & 22 & 17 \\
\hline Total & 700 & 700 & 700 \\
\hline
\end{tabular}

* Solid dispersion and physical mixture are in proportion of drug:carrier 1:3, hence $500 \mathrm{mg}$ of total formulation is equivalent to $100 \mathrm{mg}$ of drug; batch TF6, TF24 and CT are coded for tablet of batch F9, batch F27 and convention tablet respectively. 
Table 5 Data of stability study conducted for six months

\begin{tabular}{|c|c|c|c|c|c|c|c|c|c|}
\hline \multirow{2}{*}{ Batches } & \multicolumn{3}{|c|}{ \%CSE } & \multicolumn{3}{c|}{ Saturation Solubility } & \multicolumn{3}{c|}{ \%DD 15 } \\
\cline { 2 - 11 } & $\mathbf{0 m}$ & $\mathbf{6 m}$ & \%d & $\mathbf{0 m}$ & $\mathbf{6 m}$ & \%d & 0m & 6m & \%d \\
\hline F24/TF24* & 170.93 & 150.43 & -11.99 & 3.511 & 2.173 & -38.11 & 48.82 & 29.40 & -39.78 \\
\hline F6/TF6* & 162.85 & 143.65 & -11.78 & 1.229 & 1.143 & -7.00 & 29.95 & 27.32 & -8.78 \\
\hline drug/CT* & 140.56 & 131.56 & -6.40 & 1.121 & 0.936 & -16.50 & 32.89 & 27.01 & -17.88 \\
\hline
\end{tabular}

$\%$ CDR 15= Cumulative percentage drug release after 15 minutes; \% CSE and saturation solubility data is that of batch F6, F24 and pure drug; and \% CDR 15 data is of batch TF6, TF24 and CT.

\section{DISCUSSIONS}

By formulating solid dispersion of modafinil, the water solubility of pure drug was increased by 7 folds and melting point was reduced by 0 to $33 \%$ in different batches, which suggested the conversion of crystalline form to amorphous form, which was then confirmed by, DSC (Fig. 1), XRD (Fig. 2) study. While comparing different carriers, PEG6000 was found to have higher potential to enhance the water solubility of modafinil by melting method. Hence, batch F24 showing maximum solubility enhancement was selected for extensive characterisation and compared with batch F6, as it is melting method of drug and PEG6000 in the same proportion.

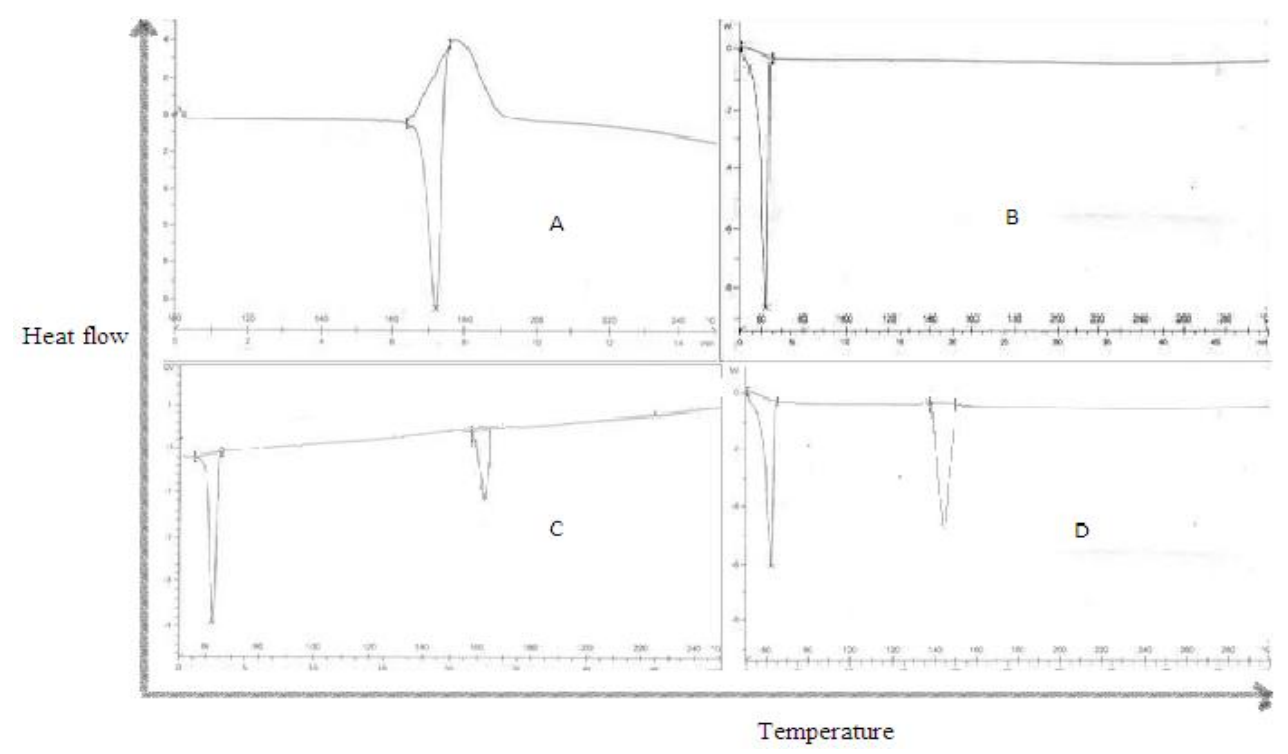

Fig. 1 DSC thermogram of A) pure drug, B) pure PEG 6000, C) batch F24 and D) batch F6

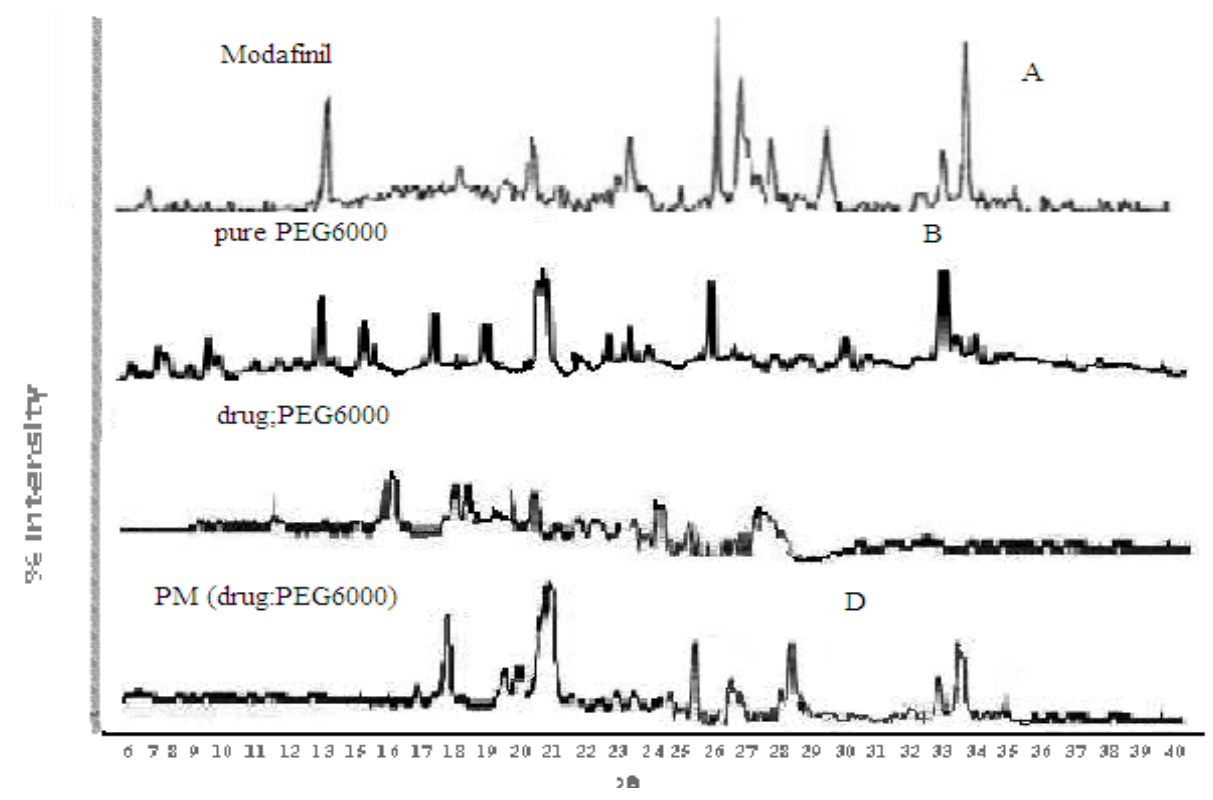

Fig. 2 X-ray diffraction spectra of pure drug (A), PEG 6000 (B), solid dispersion of drug:PEG 6000 (C) at 1:3 ratio and physical mixture of drug:PEG 6000 (D) at 1:3 ratio 
DSC thermogram of pure drug has shown very sharp melting endotherm at $166^{\circ} \mathrm{C}-171^{\circ} \mathrm{C}$ for melting and an exotherm at $174^{\circ} \mathrm{C}-190^{\circ} \mathrm{C}$ due to its decomposition. DSC thermogram of PEG 6000 has given a sharp endotherm at $59^{\circ} \mathrm{C}-62^{\circ} \mathrm{C}$ for melting. Solid dispersions batch F24 (drug:PEG6000 at 1:3 proportion by melting method) has given a sharp endotherm at $59^{\circ} \mathrm{C}-63^{\circ} \mathrm{C}$ that suggested that melting peak of drug was absent and melting point of the formulation was near to that of PEG6000. Hence the DSC study also suggested alteration in state of the drug which supported the amorphisation of drug that led to increase in solubility of the drug.

It was observed in the XRD study that pure drug is crystalline in nature showing at least three intense peaks along with several small to intermediate peaks in the spectra and pure carrier PEG6000 is semi crystalline in nature showing two intense peaks in the spectra, but solid dispersion has no any intense peaks and showing only few peaks with lesser intensity as compared to pure drug and carrier. Hence this study confirmed that was converted in amorphous state in solid dispersion batch F24 which led to solubility enhancement. In spectra of melting method batch F6, reduction of number of peaks as well as intensity of peaks was observed which confirmed only partial conversion of crystalline form to amorphous form.

In the present study, it was observed that as the proportion of hydrophilic carriers was increased, the solubility also increased which might be due to the improved wetting of drug particles by carrier as the particle surface rendered hydrophilic after coating by PEGs. Higher solubility enhancement was observed with PEG6000 than PEG8000 and PEG4000 due to some unknown cause but might be due to the similar melting behavior and crystalline properties which led to perfect solution of drug into carrier.
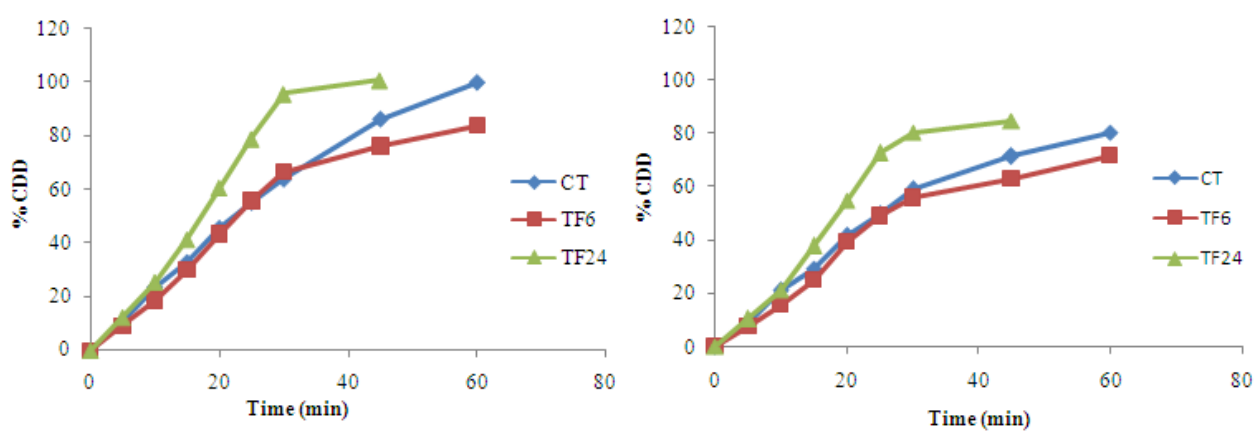

Fig. 3 Comparative in vitro drug dissolution study performed initially and after six months

Fig. 3 shows the in vitro dissolution profiles of modafinil from tablets containing solid dispersion and physical mixture of PEG6000 at 1:3 ratio; and conventional tablet. Modafinil is slightly soluble in water (16) which is reflected in the extent of drug dissolved after 15 minutes $(32.89 \%)$ from batch CT. Its hydrophobic property prevented the powder surface from contacting the dissolution medium. In contrast, the dissolution rate of modafinil from batch TF24 was significantly higher than that of batch TF6 and batch CT. The results indicated that TF24 exhibited $48.82 \%$ drug dissolution in 15 minutes as compared to only $29.95 \%$ and $32.89 \%$ from batch TF6 and batch CT respectively. The hydrophilic properties of PEG 6000 probably led to greater wetting and increased surface available for dissolution by reducing interfacial tension between the hydrophobic drug and the dissolution medium.

All the three tablets (batch TF6, TF24 and CT) were also evaluated for stability study for the duration of six months, where results of melting point, saturation solubility and one point in vitro drug dissolution study (\% cumulative drug released after 15 minutes) were compared. It was observed that, \%CSE of solid dispersion (batch F24) was increase by $11.99 \%$ as compared to $11.78 \%$ and $6.40 \%$ in melting method (batch F6) and conventional tablet (batch CT) respectively, suggested stability problem in solid dispersion. Similar results were obtained in saturation solubility study as well as in vitro dissolution study, where significant change in these properties was observed which indicated poor stability of solid dispersion or the amorphous form. Hence stabilization of solid dispersion was needed to make this formulation strategy successful.

\section{CONCLUSION}

Solid dispersions of modafinil prepared with different polyethylene glycols (PEG4000, PEG6000 and PEG8000) by melting and solvent evaporation method resulted in increased saturation solubility of drug. Based on saturation solubility study of different solid dispersion, batch F24 was selected, as it has shown better results, and formulated into tablet along with batch F6 and pure drug; and evaluated for in vitro dissolution and stability study. As demonstrated by characterization of solid dispersion by DSC, XRD study, a decreased crystallinity of modafinil as well as the surface morphology of the polymeric particles can explain the enhanced solubility and improved dissolution rate. Tablets containing solid dispersion (batch TF24) had drug dissolution profiles that were better than those of batch TF6 and batch CT which explains that solid dispersion can be utilized successfully to enhance the water solubility of poorly water soluble drug.

\section{ACKNOWLEDGEMENT}

The authors acknowledge alembic pharmaceuticals Ltd. for providing modafinil as a gift sample. 


\section{REFERENCES}

1. Chiou WL, Riegelman S. Pharmaceutical applications of solid dispersion systems, Journal of Pharmaceutical Sciences, 1971, 60(9), 1280-1302.

2. Dhirendra K., Lewis S., Udupa N. and Atin, K, Solid dispersions: A Review, Pakistan Journal of Pharmaceutical Sciences, 2009, 22(2), 234-246.

3. Vasconcelos T, Sarmento B, Costa P, Solid dispersions as strategy to improve oral bioavailability of poor water soluble drugs, Drug Discovery Today, 2007, 12(23-24), 1068-1075.

4. Craig DQM, The mechanism of drug release from solid dispersions in water soluble polymers, International Journal of Pharmaceutics, 2002, 231(2), 131-144.

5. Hancock BC, Disordered drug delivery: destiny, dynamics and the Deborah number, Journal of Pharmacy and Pharmacology, 2002, 54(6), 737-746.

6. Ahuja N, Katare OP, Singh B, Studies on dissolution enhancement and mathematical modeling of drug release of a poorly water-soluble drug using water-soluble carriers, European Journal of Pharmaceutics and Biopharmaceutics, 2007, 65(1), 26-38.

7. Vippagunta SR, Wang Z, Hornung S, Krill S, Factors affecting the formation of eutectic solid dispersions and their dissolution behavior. Journal of Pharmaceutical Sciences, 2007, 96(2), 294-304.

8. Kennedy M, Hu J, Gao P, Li L, Ali-Reynolds A, Chal B,Gupta V, Ma C, Mahajan N, Akrami A and Sekhar Surapaneni, Enhanced bioavailability of a poorly soluble vr1 antagonist using an amorphous solid dispersion approach: A case study , 2008, 5(6), 981-993.

9. Khawam A, Flanagan A, Solid-state kinetic model: basics and mathematical fundamentals, The journal of physical chemistry B, 2006, 110 (35) ,17315-17328.
10. Marsac PJ, Konno H, Taylor LS, A comparison of the physical stability of amorphous felodipine and nifedipine systems, Pharmaceutical Research, 2006, 23(10), 2306-2315.

11. Marsac PJ, Shamblin SL, Taylor LS, Theoretical and practical approaches for prediction of drug-polymer miscibility and solubility, Pharmaceutical Research, 2006, 23(06), 24172426.

12. Christian L, Jennifer D, Improving drug solubility for oral delivery using solid dispersions, European Journal of Pharmaceutics and Biopharmaceutics, 2000, 50(1), 47-60.

13. Chiou WL, Riegelman S, Preparation and dissolution characteristics of several fast-release solid dispersions of griseofulvin, Journal of Pharmaceutical Sciences, 1969, 58(12), 1505-1510.

14. Hajratwala BR, Dissolution of solid dispersion systems, Journal of Pharmaceutical Sciences, 1974, NS3, 101-109.

15. Minzenberg MJ, Carter CS, Modafinil: A Review of Neurochemical Actions and Effects on Cognition, Neuropsychopharmacology, 2007, 33, 1477-1502.

16. Jacobs MJ, McIntyre BT, Parikh A, Patel PR. Pharmaceutical solutions of modafinil compounds, U.S. Patent, 2002, 378919.

17. Barmpalexis P, Koutsidis I, Karavas E.et al, Development of pvp/peg mixtures as appropriate carriers for preparation of drug solid dispersions by melt mixing technique and optimization of dissolution using artificial neural networks, European Journal of Pharmaceutics and Biopharmaceutics, 2013.

18. Howlader IS, Chakrabarty KJ, Enhancing dissolution profile of diazepam using hydrophilic polymers by solid dispersion technique, International Current Pharmaceutical Journal, 2012, 1(12),423-430. 